\title{
Induction effects of torus knots and unknots
}

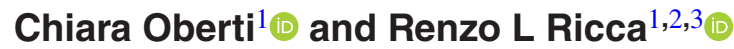 \\ ${ }^{1}$ Department of Mathematics and Applications, U. Milano-Bicocca, Via Cozzi 55, \\ 20125 Milano, Italy \\ 2 BDIC, Beijing U. Technology, 100 Pingleyuan, Beijing 100124, People's Republic \\ of China \\ E-mail: c.oberti@campus.unimib.it and renzo.ricca@unimib.it
}

Received 4 March 2017, revised 9 July 2017

Accepted for publication 19 July 2017

Published 9 August 2017

\begin{abstract}
Geometric and topological aspects associated with induction effects of field lines in the shape of torus knots/unknots are examined and discussed in detail. Knots are assumed to lie on a mathematical torus of circular cross-section and are parametrized by standard equations. The induced field is computed by direct integration of the Biot-Savart law. Field line patterns of the induced field are obtained and several properties are examined for a large family of knots/unknots up to 51 crossings. The intensity of the induced field at the origin of the reference system (center of the torus) is found to depend linearly on the number of toroidal coils and reaches maximum values near the boundary of the mathematical torus. New analytical estimates and bounds on energy and helicity are established in terms of winding number and minimum crossing number. These results find useful applications in several contexts when the source field is either vorticity, electric current or magnetic field, from vortex dynamics to astrophysics and plasma physics, where highly braided magnetic fields and currents are present.
\end{abstract}

Keywords: Biot-Savart law, torus knots, winding number, magnetic braids, topological fluid mechanics, vortex filaments, electric currents

(Some figures may appear in colour only in the online journal)

\section{Introduction}

In this paper we present an accurate analysis of geometric and topological aspects associated with the induction effects produced by steady source fields $\mathbf{j}$ in the shape of torus knots and unknots in ideal conditions. Our approach is rather general and applies to those cases where

\footnotetext{
${ }^{3}$ Author to whom any correspondence should be addressed.
} 
we need to uncurl the relationship $\mathbf{j}=\nabla \times \mathbf{B}$ (with $\nabla \cdot \mathbf{B}=0$ ) to determine the induction effects $\mathbf{B}$ due to $\mathbf{j}$. The fields $\{\mathbf{B}, \mathbf{j}\}$ can be interpreted in terms of velocity-vorticity for vortex filaments in Euler equations, magnetic field-electric current for electrostatic conductors, or vector potential-magnetic field for thin magnetic flux tubes in ideal magnetohydrodynamics. Induction effects due to rectilinear, circular or helical source fields have been widely studied in the literature, but very little is known for more complex configurations and indeed, to the best of our knowledge, very little is known on topological aspects. Yet, complex structures are present in many areas of science and technology. Toroidal and poloidal fields are common in astrophysical flows and in solar physics, where magnetic fields tend to form complex patterns in the outer space. In the solar corona, in particular, most of the magnetic flux outside sunspots is concentrated in plasma loops [6], and relations between morphological and physical properties of these structures is fundamental to model and predict solar activity [8, 24, 25]. Knotted solutions have been constructed in electromagnetism [3] and vortex torus knots have been studied computationally in superfluids [16], condensed matter physics $[15,26]$ and optics [13], and even in water by laboratory experiments [14]. In fusion devices such as tokamaks and stellarators strong electric currents are used to generate and shape magnetic fields responsible for plasma confinement, where toroidal and poloidal fields are carefully engineered and controlled in order to realize stable plasma equilibria $[4,10,12]$. In all these contexts energy and safety considerations are of paramount importance and the question of estimating physical properties (such as energy and helicity) from configurational features or emitted signals is of great interest. In communication technology, for instance, backscattering techniques have been applied to probe topological differences in physical systems $[17,30]$ and knotted antennas have been proposed to inject helicity into electro-magnetohydrodynamical plasma [28]. Magnetic fields are also used to investigate electric dispersion in conducting tissues present in the brain, heart and muscles [29] and are employed in dynamical systems theory, to analyze quasi-periodic or chaotic orbits associated with complex networks of field lines [1, 2, 19].

To provide information useful in the most varied contexts of application we consider a general source field wrapped on a mathematical torus in the shape of a torus knot or unknot and we analyze its induction effects in terms of geometric and topological information. This study is based on the computation of the Biot-Savart induction law by assuming that the physical filament has an infinitesimally small cross-section and by exploiting a standard parametrization of the curve (section 2). Field line patterns of the induced field are examined on several cross-sectional planes for a large family of knots and unknots of high topological complexity up to 51 crossings (section 3). The influence of the winding number, taken as a measure of this complexity, and the intensity of the induced field evaluated in the neighborhood of the toroidal region are examined in section 4. Energy and helicity, two fundamental physical quantities of the system, are examined and the effects of topology through winding number and topological crossing number are established both numerically and analytically (section 5). Conclusions are drawn in section 6 .

\section{Biot-Savart law for torus knots and unknots}

We consider physical knots given by a steady vector field $\mathbf{j}$ defined on a filament of negligible cross-section and centerline given by a torus knot or unknot $\mathcal{T}_{p, q}$ in $\mathbb{R}^{3}$. The physical knot given by $\mathcal{T}_{p, q}$ is thus identified by a closed curve that wraps a mathematical torus $p$ times in the longitudinal (or toroidal) direction and $q$ times in the meridian (or poloidal) direction. Loosely speaking we can say that $p$ denotes the number of toroidal coils and $q$ the number of poloidal coils. Torus knots are obtained by taking $p>1$ and $q>1$, with $\{p, q\}$ co-prime integers (figure 1(a)); torus unknots are obtained when $p=1$ or $q=1$ (figure 1(b)). These are 
(a)
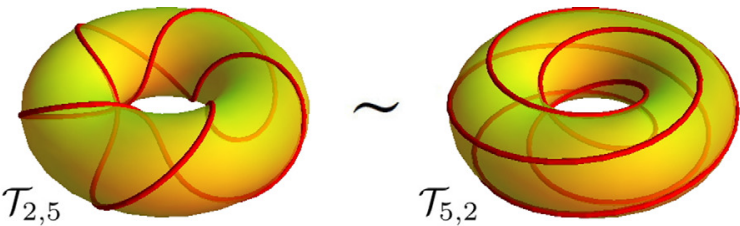

(b)
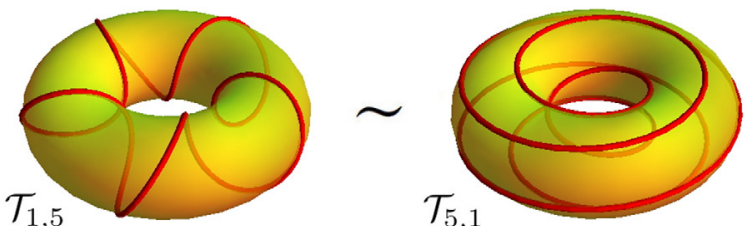

Figure 1. Torus knots and unknots (red online) drawn on a mathematical torus (yellow online). (a) The two knots $\mathcal{T}_{2,5}$ and $\mathcal{T}_{5,2}$ are the same knot type, i.e. one can be transformed into the other by continuous deformations. (b) Poloidal coil $\mathcal{T}_{1,5}$ and toroidal coil $\mathcal{T}_{5,1}$ are topologically equivalent to the standard circle (the unknot).

given by curves multiply wrapped ( $q$ or $p$ times, respectively) on the torus that are topologically equivalent to the standard circle. The ratio $w=q / p(w>0)$ is called winding number and provides a measure of the topological complexity of the knot. For fixed $p$ a poloidal hollow ring is realized in the limit when $q \rightarrow \infty$; alternatively, for fixed $q$ a toroidal hollow ring is realized when $p \rightarrow \infty$. In general, for given $p$ and $q$ the knots $\mathcal{T}_{p, q}$ and $\mathcal{T}_{q, p}$ are topologically equivalent, meaning that $\mathcal{T}_{p, q}$ can be transformed into $\mathcal{T}_{q, p}$ by a series of continuous deformations [18]; in this case we write $\mathcal{T}_{p, q} \sim \mathcal{T}_{q, p}$.

A standard parametrization of $\mathcal{T}_{p, q}$ is given by [23]

$$
\mathcal{T}_{p, q}:\left\{\begin{array}{l}
x^{*}=R(1+\lambda \cos w \alpha) \cos \alpha, \\
y^{*}=R(1+\lambda \cos w \alpha) \sin \alpha, \\
z^{*}=R \lambda \sin w \alpha,
\end{array}\right.
$$

where $\mathbf{x}^{*}=\left(x^{*}(\alpha), y^{*}(\alpha), z^{*}(\alpha)\right)$ denotes the vector position of a point on $\mathcal{T}_{p, q}$ and $\alpha \in[0,2 \pi p)$ is a parameter on the curve; $R$ and $r(0<r<R)$ denote the large and small radius of the mathematical torus, and $\lambda=r / R$ with $\lambda \in(0,1)$ the aspect ratio.

For simplicity we take a source field given by $\mathbf{j}=j_{0} \hat{\mathbf{t}}$ with $j_{0}$ constant and $\hat{\mathbf{t}}$ unit tangent to $\mathcal{T}_{p, q}$, so that the physical filament is identified with the centerline $\mathcal{T}_{p, q}$ carrying a constant flux $\Phi$. The source field $\mathbf{j}=\nabla \times \mathbf{B}$, (with $\nabla \cdot \mathbf{B}=0$ ) induces the $\mathbf{B}$-field in the exterior of the knot given by the Biot-Savart law

$$
\mathbf{B}(\mathbf{x})=\frac{\Phi}{4 \pi} \int_{\mathcal{T}_{p, q}} \frac{\hat{\mathbf{t}}\left(\mathbf{x}^{*}\right) \times\left(\mathbf{x}-\mathbf{x}^{*}\right)}{\left|\mathbf{x}-\mathbf{x}^{*}\right|^{3}} \mathrm{dx}^{*} .
$$

By using (1) the above integral reduces to

$$
\mathbf{B}(\mathbf{x})=\frac{\Phi}{4 \pi} \int_{0}^{2 \pi p} \frac{\hat{\mathbf{t}}(\alpha) \times\left(\mathbf{x}-\mathbf{x}^{*}(\alpha)\right)}{\left|\mathbf{x}-\mathbf{x}^{*}(\alpha)\right|^{3}}\left|\dot{\mathbf{x}}^{*}(\alpha)\right| \mathrm{d} \alpha .
$$

Even in this simplified form this integral is not amenable to an explicit, closed form by analytic integration. We must therefore resort to studying the influence of geometric and topological complexity by means of direct numerical integration; we shall do this in the sections below by setting $\Phi / 4 \pi=1$. 


\section{Field line patterns of the induced field}

To get an idea of the induction effects of the source field geometry and topology we first analyze the field line patterns of a given knot on different cross-sectional planes and then compare these patterns by considering different knots and unknots. To do this we proceed as follows: (i) by using (3) we compute $B_{x}, B_{y}, B_{z}$; (ii) we determine the projection $P(\mathbf{B})$ onto the desired cross-sectional plane and evaluate $P(\mathbf{B})$ at several points $\mathbf{x}$ in this plane; (iii) we plot the field lines as the envelope of $P(\mathbf{B})$. Such patterns are produced by standard routines which read twocomponent vectors only. Since in general $P(\mathbf{B})$ has three non-zero components we must make use of a standard rendering technique. In what follows we shall employ Mathematica's StreamPlot [31] which reads the vector $\mathbf{V}=\left(V_{1}, V_{2}\right) \in \mathbb{R}^{2}$; hence we can only plot field lines on the two principal planes, $x=0$ from $\mathbf{V}=\left(B_{y}, B_{z}\right)$ by using $P_{x}(\mathbf{B})=\left(0, B_{y}, B_{z}\right)$, and $y=0$ from $\mathbf{V}=\left(B_{x}, B_{z}\right)$ by using $P_{y}(\mathbf{B})=\left(B_{x}, 0, B_{z}\right)$. For any other plane we apply a rendering technique based on the use of the rotation matrix $R(\phi)$, given by

$$
R(\phi)=\left(\begin{array}{ccc}
\cos \phi & -\sin \phi & 0 \\
\sin \phi & \cos \phi & 0 \\
0 & 0 & 1
\end{array}\right)
$$

To illustrate how this procedure works consider the example given by the projection onto the plane $\Pi_{\pi / 6}$ through the $\theta=\pi / 6$ rotation of the $(x, z)$-plane $\Pi_{0}$ around the $z$-axis (see figure 2 ). We proceed as follows:

1. apply the rotation matrix (4) with $\phi=\pi / 6$ to map $\mathbf{x}=(x, 0, z) \in \Pi_{0}$ to $\mathbf{x}_{\pi / 6}=[(\sqrt{3} / 2) x,(1 / 2) x, z] \in \Pi_{\pi / 6} ;$

2. project $\mathbf{B}$ onto the plane $\Pi_{\pi / 6}$ with orthonormal basis given by $\hat{\mathbf{w}}_{1}=(\sqrt{3} / 2,1 / 2,0)$ and $\hat{\mathbf{w}}_{2}=(0,0,1)$, to get

$$
P(\mathbf{B})=\left(\mathbf{B} \cdot \hat{\mathbf{w}}_{1}\right) \hat{\mathbf{w}}_{1}+\left(\mathbf{B} \cdot \hat{\mathbf{w}}_{2}\right) \mathbf{w}_{2}=\left(\frac{3}{4} B_{x}+\frac{\sqrt{3}}{4} B_{y}, \frac{\sqrt{3}}{4} B_{x}+\frac{1}{4} B_{y}, B_{z}\right) ;
$$

3. rotate $P(\mathbf{B})$ by $-\pi / 6$ (backward) to get the components on $\Pi_{0}$ :

$$
\mathbf{B}_{\pi / 6}=\left(\frac{\sqrt{3}}{2} B_{x}+\frac{1}{2} B_{y}, 0, B_{z}\right) ;
$$

4. use StreamPlot with input given by

$$
\mathbf{V}=\left(\frac{\sqrt{3}}{2} B_{x}\left(\mathbf{x}_{\pi / 6}\right)+\frac{1}{2} B_{y}\left(\mathbf{x}_{\pi / 6}\right), B_{z}\left(\mathbf{x}_{\pi / 6}\right)\right) .
$$

As we see two rotations have been applied: a first rotation to obtain points on $\Pi_{\pi / 6}$ from points on $\Pi_{0}$, and a second rotation to reduce the non-zero components of $P(\mathbf{B})$ to two. This is a general procedure that works for any given $3 \mathrm{D}$ vector field and does not rely on any symmetry property of the system.

Distinct patterns induced by several knot types have been analyzed. Induced fields given by the knot $\mathcal{T}_{2,3}(R=1$ and $\lambda=0.75)$ on various cross-sectional planes are shown in figure 2 . The region (yellow online) interior to the mathematical torus is delimited by circles and the knot intersections with the cross-sectional plane are denoted by bullets. Given the rotational symmetry of the standardly embedded knot, the pattern is seen to rotate (non-isometrically) with $\theta$, becoming gradually self-similar with increasing $p$. 

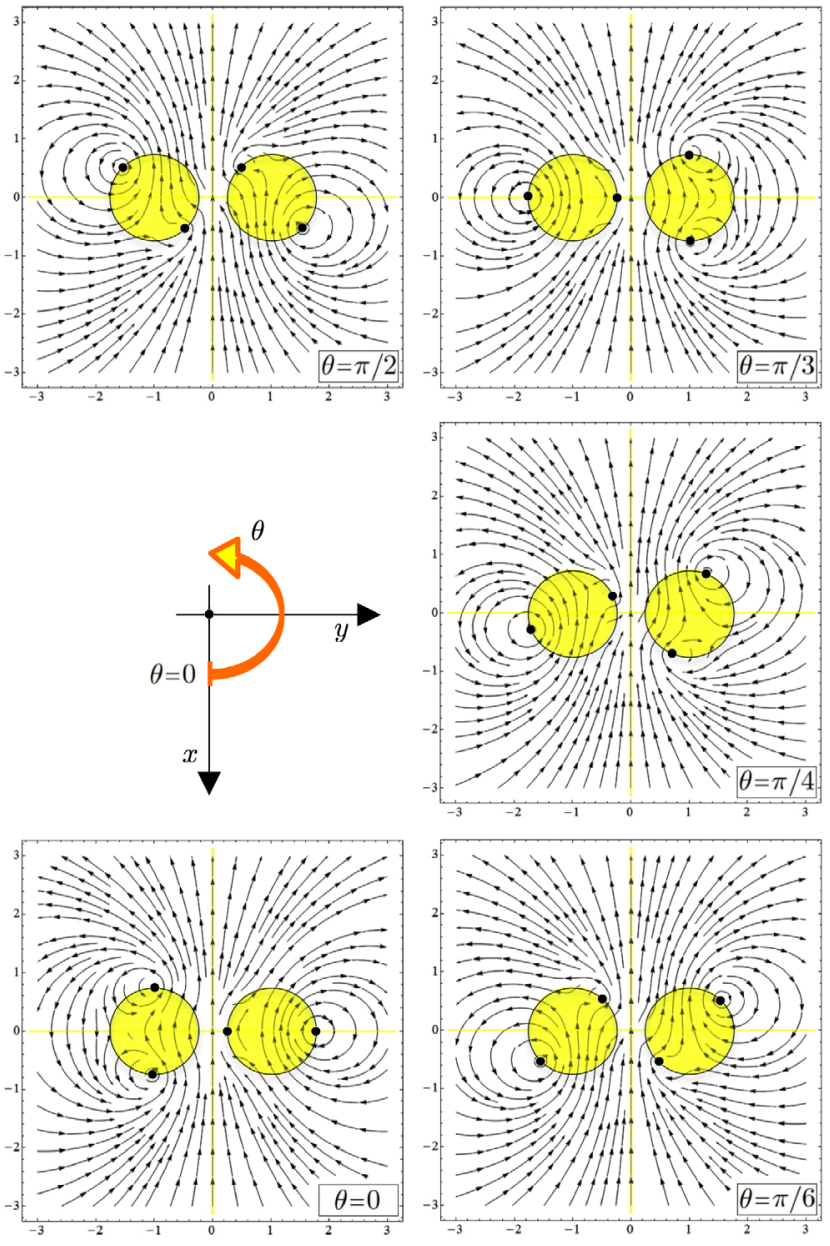

Figure 2. B-lines induced by $\mathcal{T}_{2,3}(R=1$ and $\lambda=0.75)$ plotted for increasing values of $\theta$ on cross-sectional planes $\theta=$ constant. Bullets denote the knot intersections and circles delimit the region (yellow online) interior to the mathematical torus.

Figure 3 shows for comparison the field line patterns induced by different torus knots and unknots in the plane $\theta=\pi / 6(R=1$ and $\lambda=0.75)$. At distances much greater than the average knot size the pattern becomes gradually indistinguishable from that given by a circular source field. For small $p$ (see, for example, figure 3(e)) the pattern resembles that given by $2 p$ parallel, straight field lines orthogonal to the cross-sectional plane. For large $p$ the source field becomes mainly toroidal, with the poloidal component of the induced field dominant (see figure 3(f)). As $q$ increases toroidal effects become more pronounced and they cannot be visualized on cross-sectional planes.

\section{Influence of winding number and knot type}

The influence of the winding number $w$ on the intensity $|\mathbf{B}|$, computed at different induction points on the $x$-axis, is shown in figure 4. At the origin $x=0|\mathbf{B}|$ grows with the number of toroidal wraps, remaining almost constant for a poloidal source field. When $x$ moves away 
(a)

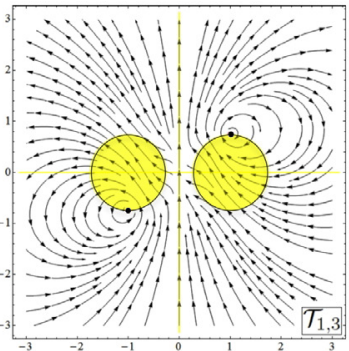

(c)

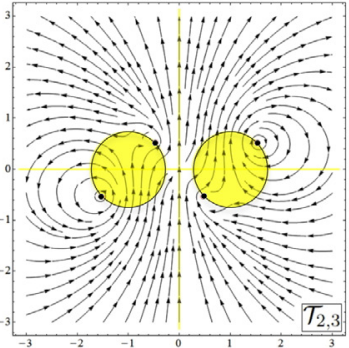

(e)

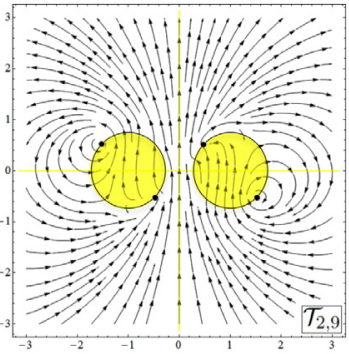

(b)

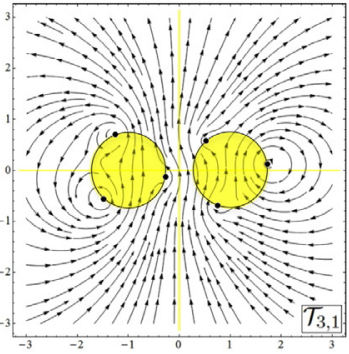

(d)

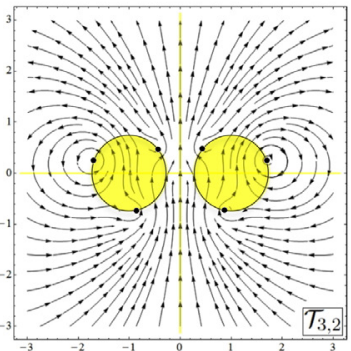

(f)

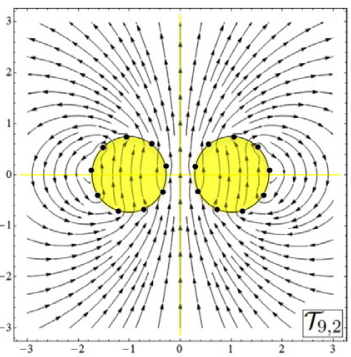

Figure 3. B-lines induced by different torus knots and unknots in the cross-sectional plane $\theta=\pi / 6(R=1$ and $\lambda=0.75)$. Bullets denote the knot intersections and circles delimit the region (yellow online) interior to the mathematical torus.

from the center, but still very close to the torus boundary (at $x=0.2$ and $x=0.3$ ) we observe a branching of the $|\mathbf{B}|$-curves for unknots $\mathcal{T}_{p, 1}$ that depends on the parity value of $p$. To understand this let us compare, for example, $\mathcal{T}_{2,1}(w=1 / 2)$ and $\mathcal{T}_{3,1}(w=1 / 3)$. From the plot of figure 3(b) we can see how the local arrangement of the inner coils of $\mathcal{T}_{3,1}$ near the point $x=0.2$ (figure 4 top right plot) determines a value of the induced field that is much lower than that given by the inner coil of $\mathcal{T}_{2,1}$, which is very close to that location. This local effect also causes the branching for points in the torus interior at $x=0.3$. More generally at $x=0.3$ poloidal source fields (i.e. with dominant poloidal geometry) induce much stronger effects with $|\mathbf{B}|$ reaching high values roughly proportional to $q$. For toroidal source fields high values are attained in the region just outside the torus between $x=0$ and $x=0.2$. As $x$ approaches the torus boundary we note a peak in $|\mathbf{B}|$, with $|\mathbf{B}| \rightarrow \mathbf{0}$ as $x \rightarrow \infty$.

Let us compute the intensity per unit length, given by

$$
|\overline{\mathbf{B}}|=\frac{|\mathbf{B}|}{L}
$$

where $L$ is the total length of $\mathcal{T}_{p, q}$. Note that $L$ increases with increasing values of $p$ and $q$. Plots of $|\mathbf{B}|$ and $|\overline{\mathbf{B}}|$ computed at $x=0$ are shown in figure 5. For knots and unknots with $q<p|\mathbf{B}|$ is proportional to the total length of the knot; since $L$ is a linear function of $p$ [23] induction 

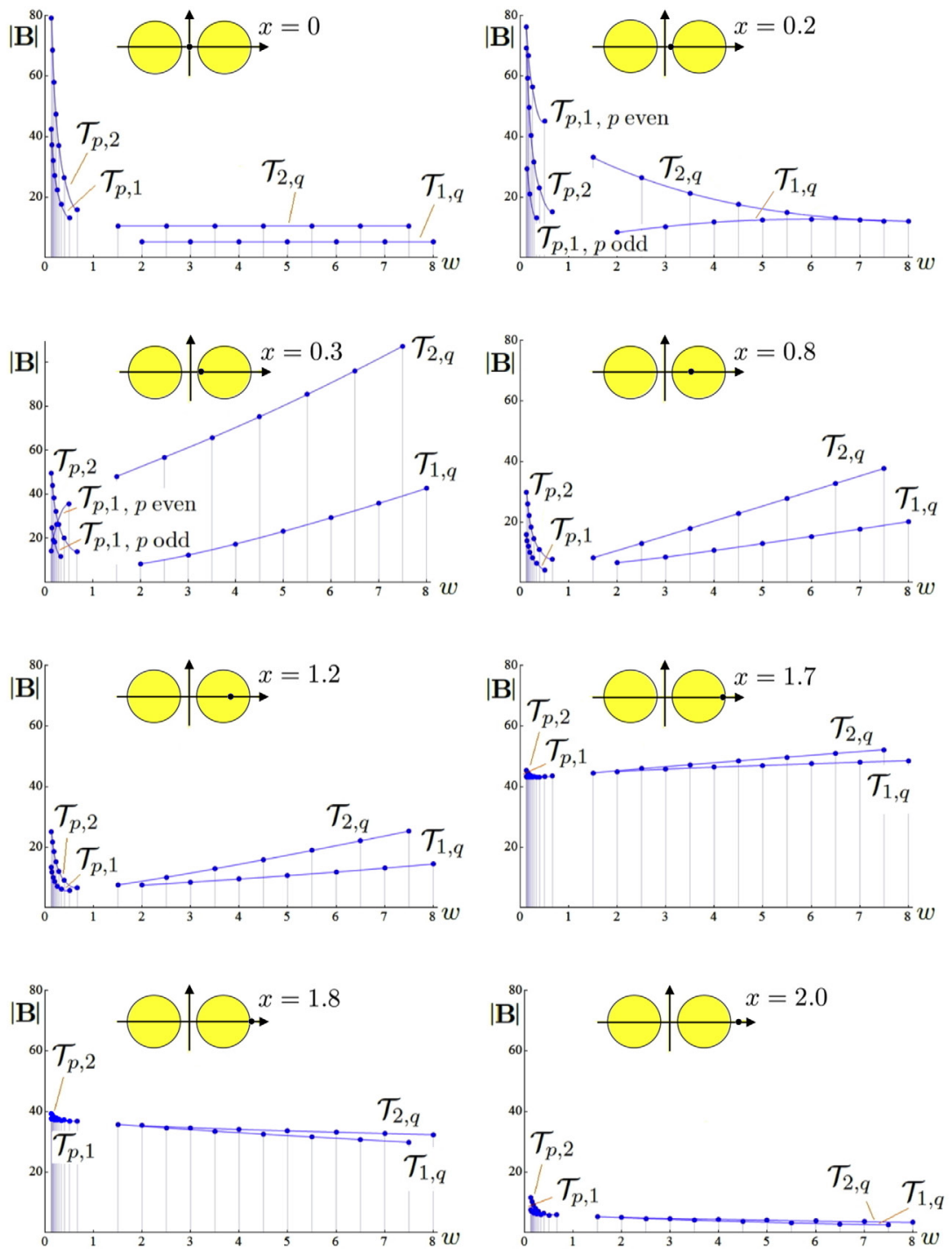

Figure 4. $|\mathbf{B}|$ against winding number $w$ for unknots $\mathcal{T}_{p, 1}$ and $\mathcal{T}_{1, q}$ with $p, q \in\{2,3,4,5,6,7,8\}$, and knots $\mathcal{T}_{p, 2}$ and $\mathcal{T}_{2, q}$ with $p, q \in\{3,5,7,9,11,13,15\}$ ( $R=1$ and $\lambda=0.75$ ). Induction points are placed on the $x$-axis. Inset: position of induction point (denoted by a bullet) with respect to toroidal region (yellow online). Interpolation is for visualization purposes only.

effects at the origin are essentially proportional to $p$ (figures 5(a) and (b), top diagrams). On the contrary, since poloidal wraps on opposite sides have opposite source field orientation, at the origin their induction effects tend to cancel out. As we can see from the plots of $\mathcal{T}_{1, q}$ and $\mathcal{T}_{2, q}$ (figures 5 (a) and (b), bottom plots) the intensity of the induced field of unknots with one toroidal coil is about half of that due to knots with two toroidal coils, being negligible the contribution from poloidal wraps.

By inspecting the functional behavior of $|\mathbf{B}|$ at various points on the $x$-axis (plots not shown here) we note that the maximum of the field's intensity remains localized near the boundary 


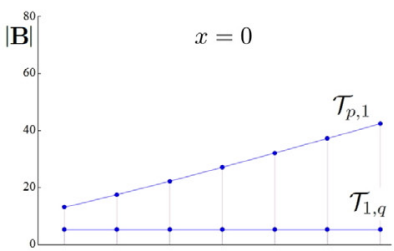

(a)

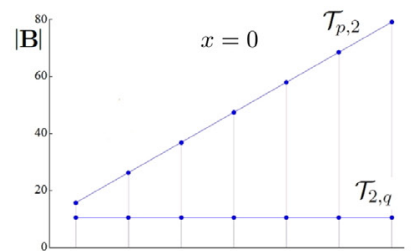

(b)

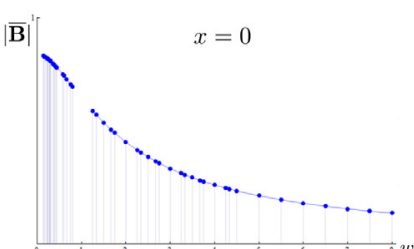

(c)

Figure 5. $|\mathbf{B}|$ and $|\overline{\mathbf{B}}|$ at the origin of the reference system $(R=1$ and $\lambda=0.75)$ (a) Unknots $\mathcal{T}_{p, 1}$ and $\mathcal{T}_{1, q}$ with $p, q \in\{2,3,4,5,6,7,8\}$; (b) knots $\mathcal{T}_{p, 2}$ and $\mathcal{T}_{2, q}$ with $p, q \in\{3,5,7,9,11,13,15\}$; knots and unknots are equally spaced on the $x$-axis. (c) Intensity per unit length $|\overline{\mathbf{B}}|$ against $w$ computed at $x=0$. Interpolation is for visualization purposes only.

of the mathematical torus independently of the geometric and topological complexity of the source field. This information is useful when we consider field lines confined to a torus (like in tokamaks) that are not necessarily closed curves but rather space-filling trajectories of a dynamical system that displays chaotic behavior. In this case information on average properties is useful for physical applications. In order to have such information in a concise way we consider the average values $\langle|\mathbf{B}|\rangle$ based on the arithmetic mean of $|\mathbf{B}|$ taken over the first seven knots/unknots of the families $\mathcal{T}_{p, 1}, \mathcal{T}_{1, q}, \mathcal{T}_{p, 2}$ and $\mathcal{T}_{2, q}$ and plot $\langle|\mathbf{B}|\rangle$ at various points along the $x$-axis (figure 6). Induction points interior to the toroidal region (yellow online) are delimited between dashed, vertical lines. The two peaks of $\langle|\mathbf{B}|\rangle$ just inside the toroidal region (respectively at $x=0.3$ and $x=1.7$, see figure 6 , left plots) are generic features of all knots $\mathcal{T}_{i, q}$ s tested ( $i$ fixed, $q>i$ ). Alternatively, for $\mathcal{T}_{p, i} \mathrm{~s}\langle|\mathbf{B}|\rangle$ reaches a maximum at about $x=1.7$, behaving differently for $x \in[0,0.3]$ according to the parity of the $i$-index.

\section{Energy and helicity}

It is interesting to investigate relationships between energy, helicity and complexity. We define the integral $J=J\left(\mathcal{T}_{p, q}\right)$ given by

$$
J=\frac{1}{2} \int_{V}\left|\mathbf{j}\left(\mathrm{x}^{*}\right)\right|^{2} \mathrm{~d}^{3} \mathrm{x}^{*}
$$

over the volume $V=V\left(\mathcal{T}_{p, q}\right)$. Evidently this reduces to the conventional form of magnetic energy $\mathcal{M}$ when $\mathbf{j}$ is the magnetic field, i.e.

$$
J \equiv \mathcal{M}=\frac{1}{2} \int_{V}\left|\mathbf{B}\left(\mathbf{x}^{*}\right)\right|^{2} \mathrm{~d}^{3} \mathbf{x}^{*} .
$$

Alternatively, when the source field is vorticity the integral (9) is enstrophy. Since the knot is very thin, $J$ can be reduced to a function of the knot length $L$. If we normalize $J$ with respect to the value $J_{0}$ of a planar, circular filament of radius $R$ and the same cross-section and strength of $\mathcal{T}_{p, q}$ we have the non-dimensional quantity $\bar{J}$ given by

$$
\bar{J}=\frac{J}{J_{0}}=\bar{L}
$$

where $\bar{L}=L / 2 \pi R$ is the non-dimensional length. Now, from elementary geometric properties of torus knots [23] we have lower and upper bounds on $\bar{J}$ given by 
$\langle|\mathbf{B}|\rangle$

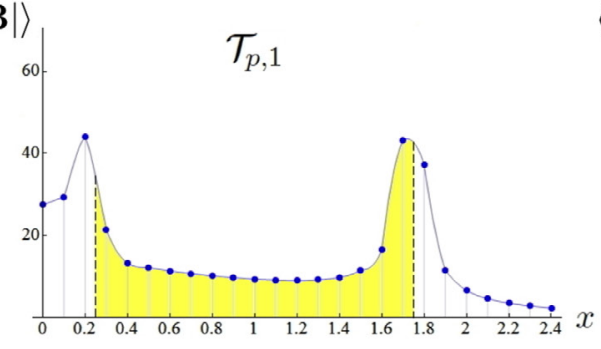

$\langle|\mathbf{B}|\rangle$

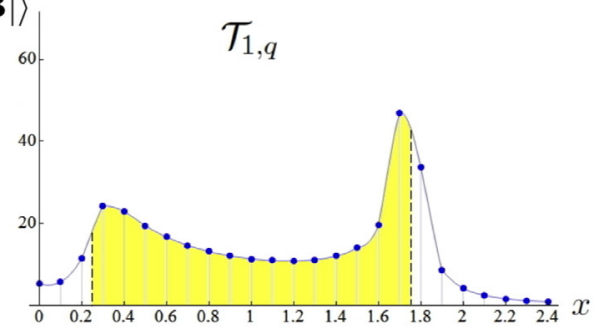

$\langle|\mathbf{B}|\rangle$

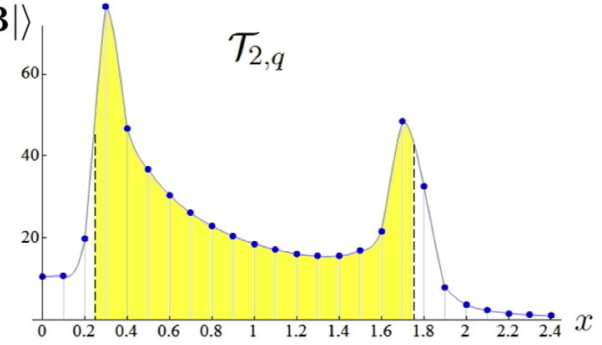

Figure 6. Mean intensity $\langle|\mathbf{B}|\rangle$ at various positions along the $x$-axis. Mean values taken over the families $\mathcal{T}_{p, 1}$ and $\mathcal{T}_{1, q}(p, q \in\{2,3,4,5,6,7,8\}$, top row $)$, and $\mathcal{T}_{p, 2}$ and $\mathcal{T}_{2, q}$ ( $p, q \in\{3,5,7,9,11,13,15\}$, bottom); $R=1$ and $\lambda=0.75$. The toroidal region (yellow online) is delimited between dashed, vertical lines. Interpolation is for visualization purposes only.

$$
p \sqrt{(1-\lambda)^{2}+\lambda^{2} w^{2}} \leqslant \bar{J} \leqslant p \sqrt{(1+\lambda)^{2}+\lambda^{2} w^{2}},
$$

and in general

$$
\begin{array}{ll}
\text { (i) } & \bar{J} \\
\text { (ii) } & \approx \lambda q \quad(q \gg p), \\
\bar{J} & \approx(1+\lambda) p \quad(p \gg q) .
\end{array}
$$

Moreover, since $p$ and $q$ are related to the topological crossing number $c_{\min }$ of $\mathcal{T}_{p, q}$ [22] by

$$
c_{\min }\left(\mathcal{T}_{p, q}\right)=\min [p(q-1), q(p-1)],
$$

i.e. $c_{\min }=q(p-1)$ if $q>p$ and $c_{\min }=p(q-1)$ if $p>q$, by (13) we have

$$
\begin{array}{lll}
\text { (i) } & \bar{J} \approx \frac{\lambda}{p-1} c_{\min } & (q \gg p), \\
\text { (ii) } & \bar{J} \approx \frac{1+\lambda}{q-1} c_{\min } & (p \gg q),
\end{array}
$$

showing that $\bar{J}$ is linearly proportional to the topological complexity of the knot. An alternative way to see the effect of topology comes from the winding number $w$ that can be related to the self-linking number $S L$ of the knot (see [23] and eqs. 18-19 below). If we take, for the sake of discussion, $S L=p q=w p^{2}=q^{2} / w$ (from 19 below) from figure 7(a) we see how topology influences the $\bar{J}$-levels for any fixed $p$ (or $q$ ).

More on this from helicity, that in ideal conditions is a fundamental topological invariant of the system [20,32]. We define $H=H\left(\mathcal{T}_{p, q}\right)$ by taking

$$
H=\int_{V} \mathbf{j} \cdot \mathbf{B} \mathrm{d}^{3} \mathbf{x}^{*}
$$




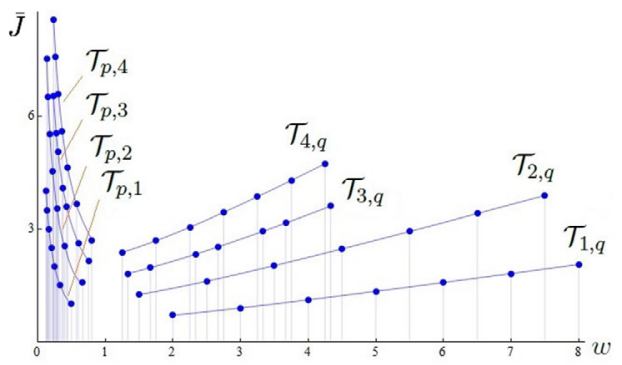

(a)

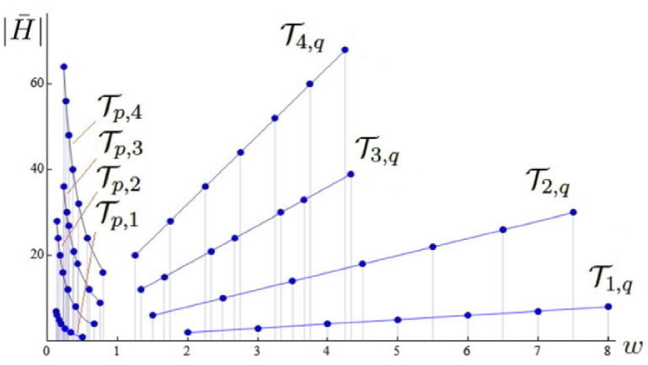

(b)

Figure 7. (a) Non-dimensional values of $\bar{J}$ and $|\bar{H}|$ against winding number $w$ for several torus knots and unknots $(R=1$ and $\lambda=0.5)$. Unknots $\mathcal{T}_{p, 1}, \mathcal{T}_{1, q}$ with $p, q \in\{2,3,4,5,6,7,8\}$; knots $\mathcal{T}_{p, 2}, \mathcal{T}_{2, q}$ with $p, q \in\{3,5,7,9,11,13,15\}, \mathcal{T}_{p, 3}, \mathcal{T}_{3, q}$ with $p, q \in\{4,5,7,8,10,11,13\}$ and $\mathcal{T}_{p, 4}, \mathcal{T}_{4, q}$ with $p, q \in\{5,7,9,11,13,15,17\}$. Interpolation is for visualization purposes only.

with $\nabla \cdot \mathbf{B}=0$. The above integral reduces to the standard magnetic helicity $\mathcal{H}_{m}$ when we take $\mathbf{j}$ to be the magnetic field, i.e.

$$
H \equiv \mathcal{H}_{m}=\int_{V} \mathbf{B} \cdot \mathbf{A} \mathrm{d}^{3} \mathbf{x}^{*}
$$

(where $\mathbf{A}$ is the associated vector potential of $\mathbf{B}$ ). Alternatively, when $\mathbf{j}$ is vorticity the integral (16) becomes kinetic helicity. $H$ can be computed by using the assumptions of section 2 and equation (3) by taking care of the logarithmic singularity that develops when source and induction points become asymptotically close to one another, in which case we need to apply a de-singularization technique (see [24]). A more practical and straightforward approach convenient to applications is given by considering the expression of helicity in terms of geometric decomposition associated with the topological interpretation of $H$ [21], given by

$$
\bar{H} \equiv S L=W r+T,
$$

where $\bar{H}=H / \Phi^{2}, S L$ is self-linking, $W r$ writhe and $T$ normalized total torsion (the field lines have no intrinsic twist). Direct computation of the geometric quantities actually shows that for these knots writhe alone would provide a rather good estimate of $\bar{H}$ [24]. An even simpler estimate of $S L$ is given by assuming that inflexions (points of zero curvature present only when $\lambda=\lambda_{\text {cr }}=\left(1+w^{2}\right)^{-1}$, see [23]) are absent, and by applying a result of Fuller $\mathrm{Jr}$ (see [23] section 5), we have

$$
|\bar{H}| \equiv|S L|=\left\{\begin{array}{lll}
q(p-1) & \text { if } \quad 0<\lambda<\lambda_{\text {cr }} \\
p q & \text { if } \quad \lambda_{\text {cr }}<\lambda<1
\end{array}\right.
$$

Plots of $|\bar{H}|$ against $w$ are shown in figure 7(b). As for magnetic energy, also helicity levels are evidently influenced by topology through $w$ for given fixed values of $p$ (or $q$ ).

\section{Conclusions}

In this paper we have carried out a detailed analysis of the influence of geometric and topological properties on the induction effects of a source field in the shape of torus knots and unknots in ideal conditions. This is done by considering the standard embedding of several knot types 
(up to 51 crossings) onto a mathematical torus. Knot types are described by a parametric representation of the curve and induction effects associated with the field, identified with a given knot, are computed by the Biot-Savart law. The pattern of the induced field lines are extracted and analyzed on several cross-sectional planes for different knot families and intensity is calculated at various locations, inside and outside the toroidal region. Geometric features associated with rotational symmetry of the standard embedding are reflected in the self-similar features of the patterns. The intensity of the induced field measured at the center of symmetry is found to grow linearly (to a first approximation) with the number $p$ of toroidal coils, remaining almost uninfluenced by the number $q$ of poloidal coils. Maxima of intensity are found in the interior region of the mathematical torus and next to its boundary, a generic feature observed for every $\mathcal{T}_{p, q}$ independently of knot complexity. The effects of local geometry are clearly dominant, but second order effects due to topology are more significant at a distance of the order of the torus cross-sectional size, becoming negligible as $|\mathbf{x}| \rightarrow \infty$, where the field's effects are indistinguishable from those given by a circular wire. These results are consistent with what has been reported in literature $[17,28]$. The effects of the winding number (which can be interpreted as a topological invariant of the system through the self-linking number) on the energy and helicity are examined in details numerically and analytically, showing that energy and helicity levels are influenced by growing topological complexity and remarkable correspondence between energy and helicity dependence on $w$ is found. Since physical knots have negligible crosssections, simple relations of energy and helicity in terms of $p, q$ and $c_{\min }$ are established, showing that both energy and helicity grow with $c_{\text {min }}$ and, depending on the value of $p$ or $q$, with $w$.

The results presented here are of general validity and find applications in a wide variety of contexts that involve field confinement in toroidal geometry or braided patterns, most notably in magnetic confinement of plasma in tokamaks and stellarators [33], or in the study of braided structures in the solar corona $[6,7]$. In the first case winding number information is important in engineering optimal confinement and efficiency of the burning plasma since $w$ is directly related to the tokamak's safety factor $\chi(\propto 1 / w)$ [9] and stellarator's rotational transform $\xi(\propto w)[11,33]$. In the second case, writhe and structural complexity measurements based on crossing number information [5, 25, 27] can help to estimate energy and helicity contents in energy build-up processes and flare predictions.

\section{Acknowledgments}

C Oberti wishes to thank the Isaac Newton Institute for Mathematical Sciences (Cambridge, UK) and the International Center for Mathematical Sciences (Edinburgh, UK) for their kind hospitality during the preparation of this work.

\section{ORCID iDs}

Chiara Oberti (1) https://orcid.org/0000-0002-8860-4823

Renzo L Ricca (1) https://orcid.org/0000-0002-7304-4042

\section{References}

[1] Aguirre J, Giné J and Peralta-Salas D 2008 Integrability of magnetic fields created by current distributions Nonlinearity 21 51-69

[2] Aguirre J and Peralta-Salas D 2007 Realistic examples of chaotic magnetic fields created by wires Europhys. Lett. 8060007 
[3] Arrayás M, Bouwmeesterb D and Trueba J L 2017 Knots in electromagnetism Phys. Rep. 667 1-61

[4] Ball J, Parra F I, Barnes M, Dorland W, Hammett G W, Rodrigues P and Loureiro N F 2014 Intrinsic momentum transport in up-down asymmetric tokamaks Plasma Phys. Control. Fusion 56095014

[5] Berger M A 1993 Energy-crossing number relations for braided magnetic fields Phys. Rev. Lett. $70705-8$

[6] Bray R J, Cram L E, Durrant C and Loughhead R E 2005 Plasma Loops in the Solar Corona (Cambridge: Cambridge University Press)

[7] Cirtain J W et al 2013 Energy release in the solar corona from spatially resolved magnetic braids Nature 493 501-3

[8] Demoulin P, Pariat E and Berger M A 2006 Basic properties of mutual magnetic helicity Solar Phys. 233 3-27

[9] Evans T E et al 2006 Edge stability and transport control with resonant magnetic perturbations in collisionless tokamak plasmas Nat. Phys. 2419-23

[10] Freidberg J P 2007 Plasma Physics and Fusion Energy (Cambridge: Cambridge University Press)

[11] Garabedian P R 2008 Three-dimensional analysis of tokamaks and stellarators Proc. Natl Acad. Sci. USA 105 13716-9

[12] Harris J H 2004 Small to mid-sized stellarator experiments: topology, confinement and turbulence Plasma Phys. Control. Fusion 46 B77-90

[13] Kedia H, Bialynicki-Birula I, Peralta-Salas D and Irvine W T M 2013 Tying knots in light fields Phys. Rev. Lett. 111150404

[14] Kleckner D and Irvine W T M 2013 Creation and dynamics of knotted vortices Nat. Phys. 9253-8

[15] Kleckner D, Kauffman L H and Irvine W T M 2016 How superfluid vortex knots untie Nat. Phys. 12 650-5

[16] Maggioni F, Alamri S Z, Barenghi C F and Ricca R L 2010 Velocity, energy and helicity of vortex knots and unknots Phys. Rev. E 82 26309-17

[17] Manuar O and Jaggard D L 2001 Knots, symmetry and scattering IEEE Trans. Antennas Propag. 49 1299-304

[18] Massey W S 1967 Algebraic Topology. An Introduction (New York: Harcourt, Brace and World)

[19] Miyaguchi T, Hosoda M, Imagawa K and Nakamura K 2011 Topology of magnetic field lines: chaos and bifurcations emerging from two-action systems Phys. Rev. E 83016205

[20] Moffatt H K 1969 The degree of knottedness of tangled vortex lines J. Fluid Mech. 35 117-29

[21] Moffatt H K and Ricca R L 1992 Helicity and the Călugăreanu invariant Proc. R. Soc. Lond. A $439411-29$

[22] Murasugi K 1991 On the braid index of alternating links Trans. Am. Math. Soc. 326 237-60

[23] Oberti C and Ricca R L 2016 On torus knots and unknots J. Knot Theory Ramifications 251650036

[24] Oberti C and Ricca R L 2017 Energy and helicity of magnetic torus knots and braids Fluid Dyn. Res. (in press) (https://doi.org/10.1088/1873-7005/aa7bcc)

[25] Pontin D I, Candelaresi S, Russell A J B and Hornig G 2016 Braided magnetic fields: equilibria, relaxation and heating Plasma Phys. Control. Fusion 58054008

[26] Proment D, Onorato M and Barenghi C F 2012 Vortex knots in Bose-Einstein condensate Phys. Rev. E 85 1-8

[27] Ricca R L 2013 New energy and helicity lower bounds for knotted and braided magnetic fields Geophys. Astrophys. Fluid Dyn. 107 385-402

[28] Rousculp C L and Stenzel R L 1997 Helicity injection by knotted antennas into electron magnetohydrodynamical plasmas Phys. Rev. Lett. 79 837-40

[29] Sarvas J 1987 Basic mathematical and electromagnetic concepts of the biomagnetic inverse problem Phys. Med. Biol. 32 11-22

[30] Werner D H 1999 Radiation and scattering from thin toroidally knotted wires IEEE Trans. Antennas Propag. 47 1351-63

[31] Wolfram Research 2010 Mathematica (Champaign, IL: Urbana-Champaign)

[32] Woltjer L 1958 A theorem on force-free magnetic fields Proc. Natl Acad. Sci. USA 44 489-91

[33] Xu Y 2016 A general comparison between tokamak and stellarator plasmas Matter Radiat. Extremes 1 192-200 